\title{
Photonic crystals for light-emitting devices
}

\section{Thomas F. Krauss, Dominique Labilloy, Axel Scherer, Richard M. De La Rue}

Thomas F. Krauss, Dominique Labilloy, Axel Scherer, Richard M. De La Rue, "Photonic crystals for light-emitting devices," Proc. SPIE 3278, Integrated Optic Devices II, (12 January 1998); doi: 10.1117/12.298214 


\title{
Photonic crystals for light-emitting devices
}

\author{
Thomas F. Krauss ${ }^{\mathrm{a}, \mathrm{b}}$, Dominique Labilloy ${ }^{\mathrm{c}}$, Axel Scherer ${ }^{\mathrm{a}}$ and Richard M. De La Rue ${ }^{\mathrm{b}, \mathrm{d}}$ \\ ${ }^{a}$ California Institute of Technology, MS 200-36, Pasadena, CA 91125, USA. \\ ${ }^{\mathrm{b}}$ Permanent address: Optoelectronics Research Group, Department of Electronics and Electrical \\ Engineering, University of Glasgow, Glasgow G12 8QQ, Scotland, U.K. \\ ${ }^{c}$ Laboratoire de Physique de la Matiere Condensee, Ecole Polytechnique, URA 1254 CNRS, 91128 \\ Palaiseau Cedex, France. \\ ${ }^{d}$ Communications Research Lab, Nukuikitamachi 4-2-1, Koganei, Tokyo 184, Japan
}

\begin{abstract}
Photonic crystals or photonic bandgap (PBG) structures promise to revolutionise optoelectronics by making a new class of highly efficient, low noise light emitters possible. We present data to show that their properties, in particular 2-D systems, have now been fully characterised in the relevant semiconductor material system and at near-infrared wavelengths, so effort can be redirected towards making active light emitters. As a first example, we present a semiconductor laser with one output mirror designed according to PBG principles. From threshold and efficiency data, we derive a reflectivity of $95 \pm 10 \%$ for this mirror, which underlines the viability of the PBG approach for practical devices. In order to realise the full potential of photonic crystal light emitters, however, important material issues need to be considered. Non-radiative recombination, for example, is a big problem when the photonic crystal is an integral part of the active region because of the relatively large areas of exposed surface. Several possible solutions to this problem are presented.
\end{abstract}

Keywords: Photonic bandgap, Photonic microstructure, Photonic crystal, Bragg grating, Semiconductor laser, LED, Surface recombination velocity, Optoelectronics

\section{INTRODUCTION}

Photonic microstructures or photonic crystals are periodic dielectric structures designed to influence the behaviour of photons in much the same way that the atomic structure of a natural crystal affects the properties of electrons. In particular, photonic crystals forbid propagation of photons within a certain range of energies (known as the photonic bandgap, PBG), which can either be used to trap light inside the crystal ('photonic box') or to stop it from entering ('photonic reflector'). The photonic crystal's capability of trapping light is unique in the material world, because metals, despite their high reflectivity, are lossy and therefore not as useful to contain optical radiation - it would simply decay away. By trapping, i.e. localising light, the luminescence properties of the material can be fundamentally altered ${ }^{1}$, which leads to improved directionality, shorter luminescence decay time and lower noise, i.e. properties that are very desirable for communication systems (speed, noise) and light sources (brightness) in general. Both the functions of trapping and reflecting light have now been demonstrated in the near-IR regime. Discussing these first demonstrations and considering the practical issues of fully realising the potential of photonic crystals for light-emitting devices is the purpose of this paper.

\section{PASSIVE PROPERTIES}

Before confronting an active light emitting material with the prospect of being inserted into a photonic crystal, it is instructive to study the passive properties of the crystal itself. The characterisation method employed by most researchers is the transmisson measurement, which was also used to first indicate the existence of a photonic bandgap in the microwave regime $^{2}$. Although transmission measurements alone do not give rigorous proof of a PBG, they can give strong evidence for its existence. In the optical regime, mostly one and two-dimensionally periodic structures (1 and 2-D PBG) have been investigated ${ }^{3-6}$. Fig. 1 shows the transmission through a 2-D photonic 'honeycomb' lattice consisting of holes etched into a semiconductor waveguide structure ${ }^{3}$. 
(a) $\begin{array}{lll}0 & 0 \\ 0 & 0 & 0 \\ 0\end{array}$

Real space lattice

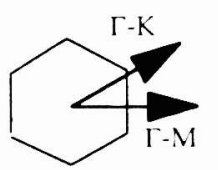

Brillouin zone with symmetry directions
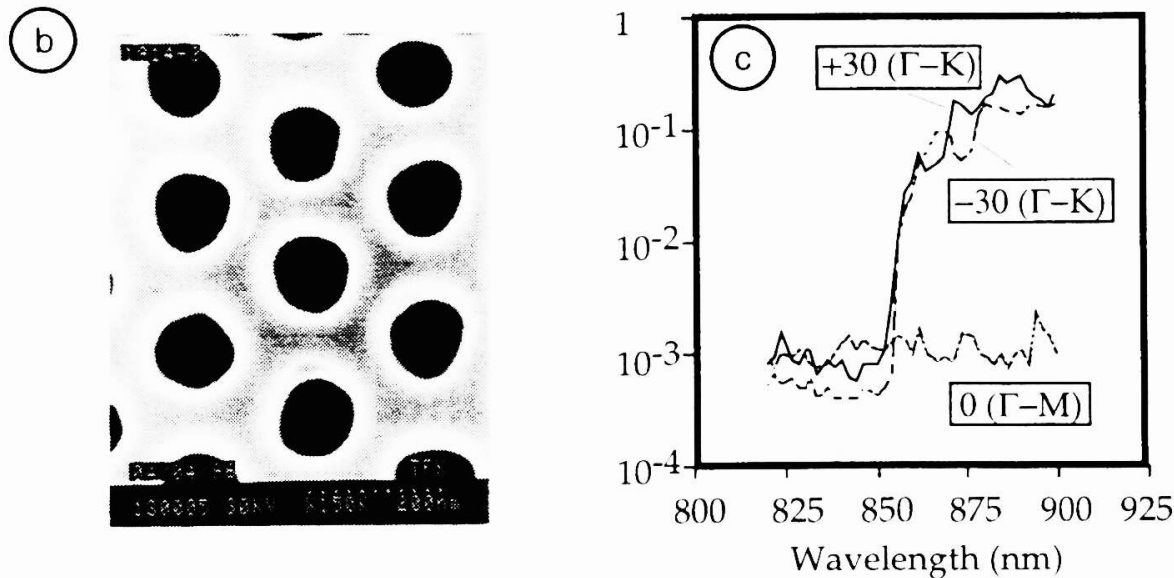

Figure $I$ a) Sketch of the real space lattice and the Brillouin zone, to illustrate the symmetry directions in the hexagonal close packed (hcp) lattice. b) Scanning electron micrograph of the lattice, which is a 'honevcomb' lattice of circular holes etched into semiconductor. The centre-to-centre spacing is $190 \mathrm{~nm}$. c) Transmission measurement on the photonic lattice (TE polarisation) showing a sharp transition from rejection (below $850 \mathrm{~nm}$. "photonic bandgap') to transmission (above $860 \mathrm{~nm}$, photonic passband'), indicating a 'photonic band-edge'. The close overlap between the two curves for $\Gamma$ - $K$. which were taken after $60^{\circ}$ rotation, indicates the almost perfect symmetry of the lattice. Note the large dynamic range of the measurement indicated by the logarithmic scale.

The data in Fig.lc was obtained by scanning the wavelength of a Ti:Sapphire laser and feeding the light into the lattice via an optical waveguide. A related approach, where the probing beam is directly generated on the wafer by photoexcitation has since been used to provide a considerably more complete picture of the photonic crystal properties ${ }^{5.7}$. The results are shown in Fig. 2 and the measurement method in Fig.3.

Figure 2 Data for transmission (a), reflection (b) and diffraction (c) of a 2-D photonic lattice, similar to that shown in Fig. Ib. for one polarisation (TE). Note the log-scale for the transmission and reflection merasurements and the linear scale for the diffraction measurement. The horizontal scale is in units of lattice period wavelength, which allows to show measurements taken on different lattices on the same graph. The diffraction data is only shown for the case of reflected light, because the diffraction of the transmitted signal is relatively weak in this case $(<10 \%)$. The axis is inverted with respect to that used in Fig. Ic; the data shown in Fig. $1 c$, on the same scale, extends from $u=0.23$ to $u=0.21$ and shows the same band-edge in $\Gamma$ - $K$ direction around $u=0.22$.

Fig. 2a essentially confirms the result first shown in Fig. $1 \mathrm{c}$, but additionally shows the full extent of the bandgap for both principal propagation directions.

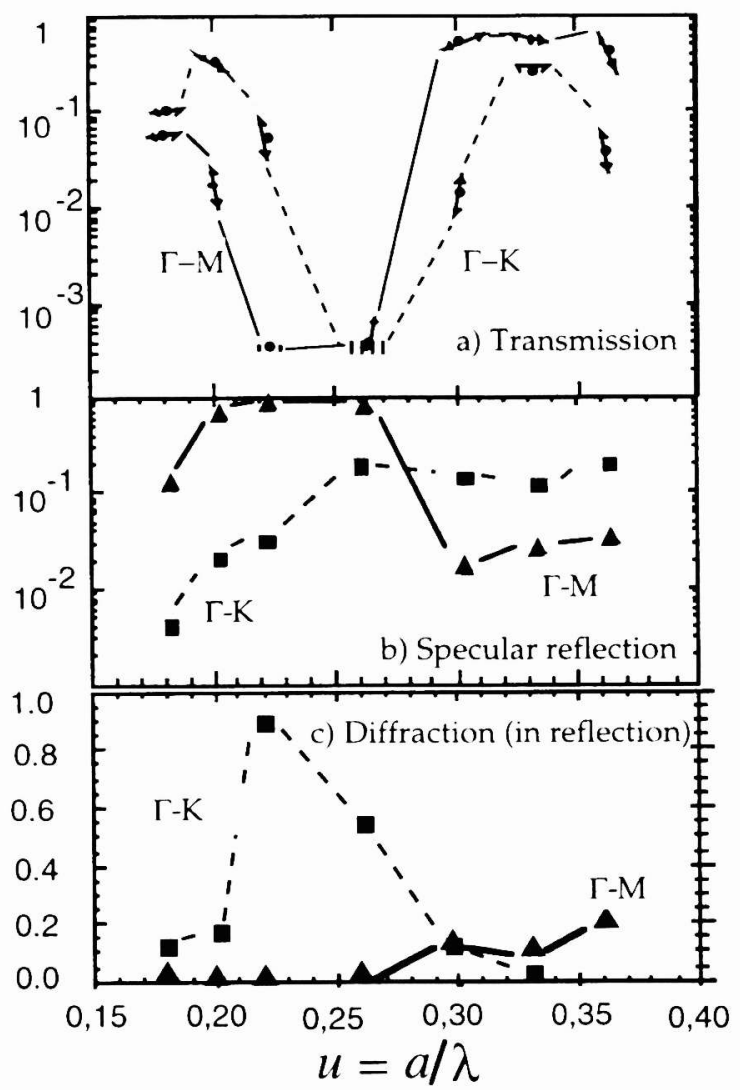

The first remarkable feature is that the regime of low transmission in the $\Gamma-\mathrm{M}$ direction corresponds to the regime of high reflection, proving that light is not scattered away on interaction with the photonic crystal. The second remarkable feature is that this correspondance fails for light propagating along the other crystallographic direction $(\Gamma-K)$. The reason for this deviation is in-plane diffraction (Fig. 2c). which is particularly strong around the low-energy band-edge at $u=0.227$. In-plane diffraction refers to the type of diffraction where the light remains confined to the waveguide plane but changes its direction of travel. In-plane diffraction must be seen in contrast to out-of-plane diffraction, which is due to fabrication imperfections, the 
limited depth of the structure and the more intrinsic lack of waveguiding in the holes. Out-of plane diffraction leads to scattering of light into the substrate and airspace above the waveguide and contributes to the optical loss of the structure.

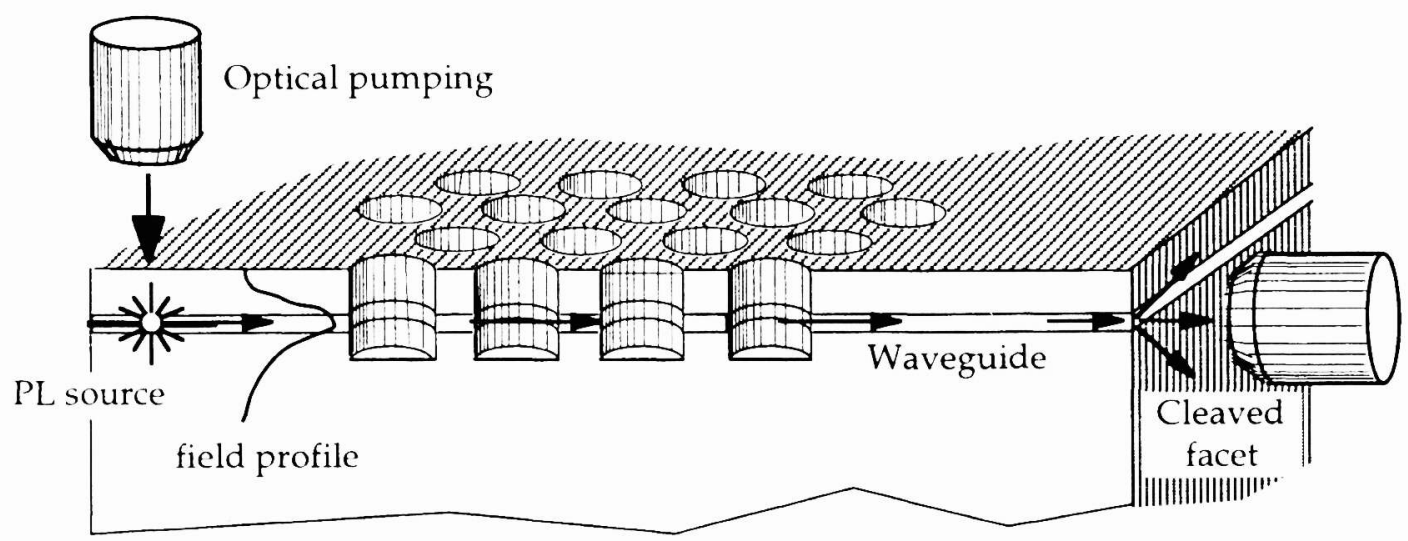

Figure 3 Sketch of the measurement scheme used to obtain the data in Fig. 2.

\section{APPLICATION 1: PHOTONIC CRYSTAL LASER MIRRORS}

One of the first applications of photonic crystals in light emitting devices is that of mirrors for semiconductor lasers, taking advantage of the high reflectivity of the PBG. When designing such a device, the question arises of what type of photonic microstructure to use, since both $1-\mathrm{D}^{4}$ and $2-\mathrm{D}^{7}$ (Fig 2) structures show high reflectivity when properly designed. The problem with 2-D structures is that of in-plane diffraction, which effectively contributes to loss for a device with a single reflector. We suspect that this diffraction phenomenon has led to the lower-than-expected performance of lasers with 2 -D mirrors realised previously ${ }^{8}$. On the other hand. 2-D mirrors are more versatile, e.g. for mode control $^{9}$ or for interaction between different elements in an integrated circuit, where the diffracted beams can be used for feedback control or in a masterslave laser arrangement. Also, if the active region is shrunk down and totally surrounded by the 2-D mirror, the diffracted light is entirely contained within the system and no longer constitutes a loss.

In the case of a relatively wide laser stripe with a single microstructured facet that only requires reflection for incidence from a single direction, however, it seems more natural to chose a 1-D periodic microstructure, which effectively eliminates the inplane diffraction problem. This type of microstructured facet, from a different perspective, can also be seen as an extreme case of the type of Bragg grating commonly used in distributed feedback (DFB) and distributed Bragg reflector (DBR) lasers. The key difference is the effective refractive index contrast. which is typically less than $1 \%$ in a DFB/DBR laser, but as large as $3.5: 1$ in the case of a photonic microstructure. This large refractive index contrast leads to a very much shorter interaction length of around $1 \mu \mathrm{m}$, instead of the $100 \mathrm{~s}$ of $\mu \mathrm{m}$ typical for a conventional DFB/DBR laser, and thereby opens the opportunity to create edge-emitting laser elements with very small optical volume.
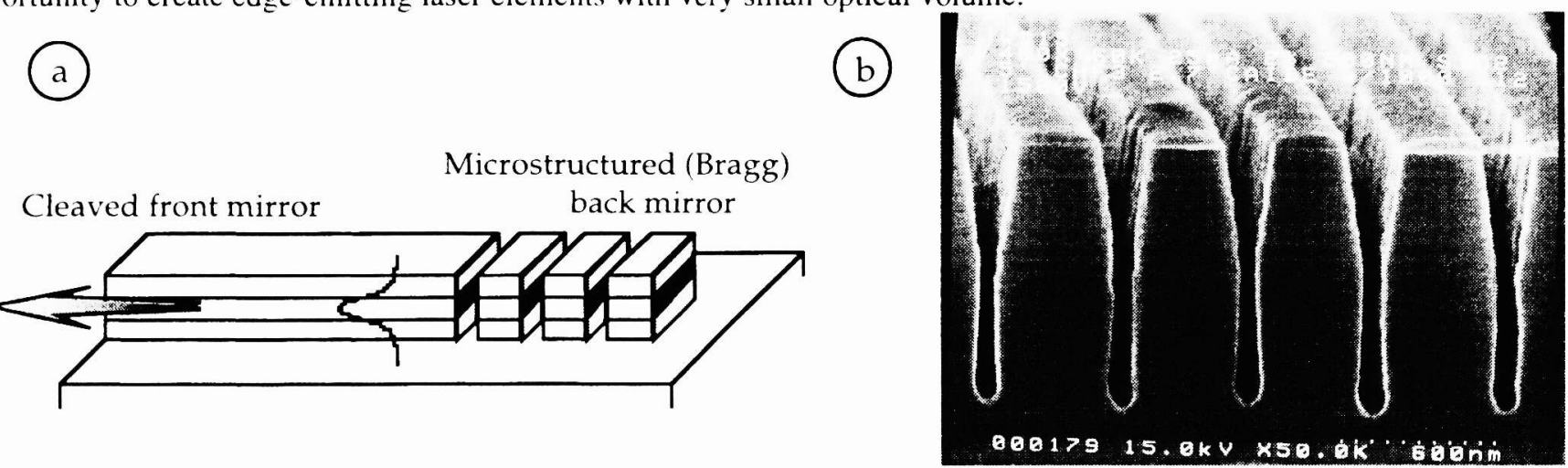

Figure 4 a) Sketch of the cavity with one microstructured and one cleaved facet. b) Cross-section of a photonic microstructure mirror. The etch depth is I $\mu \mathrm{m}$ and the quantum well region is approximately in the middle of the structure, i.e. $0.5 \mu \mathrm{m}$ below the surface. The width of the etched slots is between $60 \mathrm{~nm}$ and 100 nm and the period of this third-order grating is $500 \mathrm{~nm}$. 
a) $88 \mu \mathrm{m}$ long devices

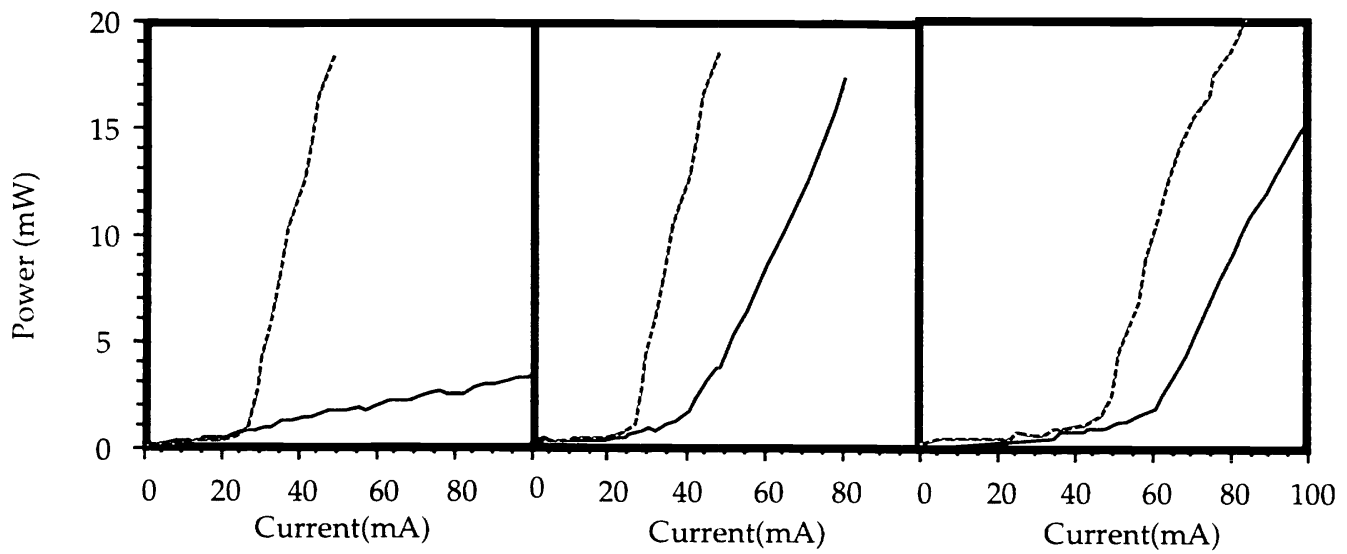

Figure 5 Light-current (L-I) curves for lasers with lengths $88 \mu \mathrm{m}($ a), $170 \mu \mathrm{m}$ (b) and $350 \mu \mathrm{m}$ (c) for both dry-etched (solid line) and microstructured (broken line) back mirrors; the front mirror is a cleaved facet in all cases. The difference between the two types of back mirror is largest for the shortest devices (a), where the mirror loss is too high for the dry-etched device to achieve lasing threshold. The difference in threshold is smallest in (c), because the internal loss, which is equal in both types of device, begins to approach the mirror loss. The width of the devices in all cases is $43 \mu \mathrm{m}$.

By comparing the performance of lasers with microstructured mirrors to that of lasers with simple etched mirrors (Fig.5) we derived a value of $95 \pm 10 \%$ for the reflectivity of the microstructured mirror ${ }^{10}$. This is a much higher value than was obtained for previously published devices ${ }^{8,11}$ and shows the possibility of using lithography to produce high-reflectivity waveguide mirrors, replacing the more cumbersome approaches of cleaving and coating typically used to date.

\section{APPLICATION 2: PHOTONIC CRYSTAL LED'S}

Light emitting diodes (LEDs) have an even greater potential for improvement than lasers, considering that typical off-the shelf devices convert no more than $3-5 \%$ of the input energy into useful light. The remaining $95-97 \%$ of the light never manages to escape from the high index material, but instead is reabsorbed and eventually dissipated as heat. A possible remedy for this problem is to modify the heart of the light generation process, by redirecting the spontaneous emission itself within the material. By enhancing emission into the wanted directions and suppressing emission into the unwanted ones, the flow of light may be radically altered and the majority of the input energy converted into useful light. One example for this strategy is the planar microcavity light emitting diode (MCLED) where the active layer is enclosed by a metallic and a DBR mirror. By reducing the number of modes allowed in the cavity, an extraction efficiency of $23 \%$ was obtained ${ }^{12}$. A very simplified but effective Fabry-Perot analysis shows that this cavity supports the equivalent of five modes, each of which captures about the same $20 \%$ fraction of the total emission, which is roughly what radiates into the single escaping mode.

The holy grail of microcavity LED's, however, is a single mode device, or a device where emission into a single mode is favoured to the extent that emission into the other modes becomes negligible. One such device has recently been described by J.-M. Gerard and colleagues at CNET, Bagneux, France. ${ }^{13}$ Its performance can best be described with the Purcell-factor, which was first introduced in $1946^{1}$, and states that the "spontaneous emission probability is ... increased, and the relaxation time reduced, by a factor"

$$
f=\frac{3}{4 \pi^{2}} \frac{Q \lambda^{3}}{V n^{3}}
$$

$\mathrm{Q}$ is the quality factor of the mode in question, $\mathrm{V}$ the mode volume and $\lambda / \mathrm{n}$ the wavelength in the material. The researchers at CNET calculated a factor of 4 for their cavity. The fact that the concept really works was then demonstrated by timeresolved measurements; when light was generated inside the structure, it radiated four times faster than it would have done without the cavity, exactly as predicted ${ }^{14}$. J.-M. Gerard concentrated on the Q-part of the equation, i.e. he designed a system with a high $\mathrm{Q}$ in order to get a high $\mathrm{Q} / \mathrm{V}$ ratio, using a quantum $\operatorname{dot}^{15}$ active layer to provide a high-Q light source.

A device recently presented by a group at MIT $^{6}$ illustrates an alternative approach: They concentrated on the other side of equation $\{1\}$, i.e. the small volume, and managed to obtain a volume of 5 cubic half-wavelengths $($ at $\lambda=1.54 \mu \mathrm{m})$, which is 
claimed to be the smallest mode volume obtained to date for a cavity in the infrared wavelength regime. Their cavity was passive, so they could not perform the same measurement as above, but they calculated a Purcell-number of 34 for their structure, which illustrates how far the concept can be pushed.

In order to make devices with such small active volumes, the luminescent properties of the material need to be considered. The majority of devices proposed so far uses some variety of etching holes or slots into the semiconductor and takes advantage of the high refractive index difference (3.5:1) between semiconductor and air. This creates the problem of nonradiative recombination at the exposed surface, which competes strongly with the desired radiative recombination in the material. Possible solutions to this problem are discussed in the following section.

\section{MATERIAL ISSUES}

The real promise of photonic microstructures arises from the fact that they can be used to control the emission process within the material in a fundamental way. In order to realise this potential, the microstructure needs to be an integral part of the active region, which incurs major problems for the luminescence properties of the material. From years of research on electronic devices with exposed surfaces, particularly in the popular GaAs/AlGaAs material system, it is known that the exposed surface of the semiconductor leads to substantial nonradiative surface recombination velocity (SRV) and reduced device lifetimes. Numerous methods have been devised to combat this problem, the most successful being based on Sulphurbased compounds, whereby the Sulphur occupies the dangling surface bonds and avoids oxidation. The sulphur can be applied in solution $\left(\mathrm{Na}_{2} \mathrm{~S},\left(\mathrm{NH}_{4}\right)_{2} \mathrm{~S}\right)$ or as a dielectric film ${ }^{16}$, the latter variation having more commercial promise than the former because it lasts longer. Just to mention one of the many passivation techniques explored more recently, it has been shown that exposing the surface of GaAs to phosphine in an MOCVD reactor leads to the formation of a stable layer of GaP, which has much more favourable properties than an untreated surface of $\mathrm{GaAs}^{17}$. The long-term stability and commercial viability of any of these techniques, however, remains to be established.

Materials science offers several alternatives to surface passivation for the photonic bandgap device engineer:

\subsection{Overgrowth}

The most obvious solution is that of overgrowing the structure with a thin (e.g. $10 \mathrm{~nm}$ ) layer of high bandgap insulating material to provide an encapsulating coating. Alternatively, the active layer can be overgrown altogether and the photonic crystal be etched into the 'passive' overgrown area similar to the way high-performance semiconductor lasers are typically produced commercially (e.g. buried heterostructure $(\mathrm{BH})$ lasers used in telecommunications). This approach is less elegant as it involves multiple growth steps and less attractive for commercial applications as it increases cost. It is well proven, however, and may also outperform the other methods presented below.

\subsection{Materials with low surface recombination velocity (SRV)}

The main problem with GaAs and its related compounds is that of a very high SRV (typically in the range $10^{5}$ to $10^{6} \mathrm{~cm} / \mathrm{s}$ ), which competes so strongly with the desired radiative recombination, that, for active areas in the sub-micron range, the luminescence efficiency drops by orders of magnitude compared to the as-grown case.

Indium-containing compounds have much more favourable properties in this respect, values as low as $600 \mathrm{~cm} / \mathrm{s}\left(\mathrm{InP} \mathrm{P}^{18}\right)$ or $\left.250 \mathrm{~cm} / \mathrm{s}_{(\mathrm{InGaAs}}{ }^{19}\right)$ have been observed. Hence, InGaAs/InP heterostructures should be superior to GaAs/AlGaAs ones. Potential problems arise from the fact that the SRV is very sensitive to various processing conditions: The damage caused by dry etching, strain caused by the deposition of thin films or heat-treatment are reasons for the SRV to increase dramatically into the $10^{4}-10^{5} \mathrm{~cm} / \mathrm{s}$ - range.

\subsection{Self-organised quantum dots}

Materials made using the "Stranski-Krastanov" growth mode ${ }^{15,20}$, where a thin, strongly-strained layer is deposited and relaxes into small islands during growth, offer another alternative to control the SRV. Typically, InAs is deposited onto GaAs. Because of the large lattice mismatch $(7 \%)$, the thin InAs film begins to break up after the deposition of approximately 2 monolayers and forms an array of InAs islands on top of the first monolayer of InAs ('wetting layer'), which remains intact. Deposition of additional GaAs freezes the island formation and encapsulates the structure. The process can be repeated a number of times, thus producing a substantial volume of efficiently luminescent material ${ }^{21}$.

Injected charge carriers in this structure are able to migrate through the wetting layer. Due to strain-assisted drift ${ }^{20}$, however, carriers will soon find themselves trapped by the quantum dot with a typical confinement energy of 100-120 meV. Therefore, the carrier diffusion length and the likelihood of recombining non-radiatively at a nearby surface are strongly reduced. A quick look at the Fermi-Dirac distribution function allows a quantitative estimate of the strength of this effect. The probability for a 
carrier to occupy a state of energy $E$ well above the Fermi level $E_{\mathrm{F}}$ is proportional to $\exp \left(\frac{E-E_{F}}{k T}\right), k T$ having its usual meaning of thermal energy. Hence, the difference of occupation between two levels $E_{1}$ and $E_{2}$ in the same system is given by $\exp \left(\frac{E_{1}-E_{2}}{k T}\right)$. From this simple model and considering that $k T$ is $25 \mathrm{meV}$ at room temperature, the presence of the dots should result in a factor of 50-100 improvement in the radiative recombination rate, or a reduction by a similar factor in the surface recombination rate. As the formula shows, the effect is more pronounced at lower temperature and almost perfect carrier capture can be achieved. This was demonstrated by J.-M Gerard and co-workers at CNET, Bagneux, France ${ }^{22}$, who showed that at $77 \mathrm{~K}$, the photoluminescence intensity of quantum dot layers grown on 'heavily dislocated' GaAs grown on Si substrates was virtually identical to that of dots grown on usual 'dislocation-free' GaAs substrates, despite the very high dislocation density resulting from the stronglty mismatched (7\%) growth of GaAs on Si. This is in strong contrast with standard quantum wells whose luminescence collapses in the GaAs-on-Si case.

\subsection{Selective oxidation}

The fourth possibility we see of tackling the surface recombination problem is to replace the semiconductor-air interface by a semiconductor-oxide interface. Selective oxidation in the GaAs/AlGaAs system is in the position to become a wellestablished process and has been used succesfully to provide current apertures in semiconductor lasers. An analysis of the SRV of this interface ${ }^{23}$ suggests a very low value, although an insufficient number of data points was presented to support a strong claim.

Most selective oxidation is carried out in the horizontal direction, where a layer with high Al-content is oxidised and layers with low Al-content are left unchanged. Recently, oxidation in the vertical direction has also been demonstrated ${ }^{24}$, where the strong dependence of the oxidation rate on aluminium content was exploited. This shows that many different design concepts can now be realised by careful control of the exact layer composition, although a suitable material design may impose severe constraints on other desired properties.

\subsection{Silicon-based light emission}

Solution number five is motivated by a different philosophy: So far, we have assumed that the material in question is a III-V semiconductor, a material family known for its highly efficient luminescent properties. The penalty for using most of these materials is the high SRV, but they also make it possible to turn a good light emitter into an exceptional one.

How about using photonic crystals to turn a bad emitter into a good one ? Silicon is known for its exceptionally low SRV $(<1 \mathrm{~cm} / \mathrm{s})^{19}$ but also, being an indirect semiconductor, for poor luminescence properties. The efficieny of electroluminescent devices made in porous or Er-doped $\mathrm{Si}$ is still well below and may not reach that of, e.g. GaAs due to fundamental limitations ${ }^{25,26}$. By employing a photonic crystal, however, to control the light emission process within the device, the luminescence efficiency may be improved considerably. This could lead to truly efficient light sources based on silicon, which would have an enormous commercial impact considering the size of the silicon industry.

\section{CONCLUSION}

In the last few years, we have learned how to make and characterise photonic crystals that are based on semiconductor waveguides. We have learned how to make periodic structures with feature sizes around $100 \mathrm{~nm}$ and how to assess their properties, as far as transmission, reflection and diffraction are concerned. The agreement with theory and the consistency between different measurements gives us confidence that the waveguide-based approach closely resembles the ideal case of infinitely deep strucures.

The first application of this knowledge was in a semiconductor laser that uses photonic microstructure mirrors. The device confirmed the high reflectivity that can be obtained with a photonic crystal if it is designed properly, a result that had hitherto only been achieved with passive test-structures. By taking this concept further, one can imagine a device where the active stripe is completely surrounded by microstructured material to effectively suppress a large part of the emission that is not directed into the lasing mode; addition of a Bragg mirror underneath the lasing stripe could suppress radiation into the substrate. The big advantage of such a device would be not so much in low threshold operation, although the low $\mathrm{mA}$ regime appears to be realistic, but more in the suppression of noise - if the spontaneous emission into the non-lasing modes is suppressed, the noise is reduced to an equal extent. Such a device would be a 'hybrid', i.e. a combination of structures that are perodic in different directions to give a quasi-3D periodic system. It would be unlikely to have a full 3D bandgap, but offer more flexibility in device design than a true 3-D photonic crystal. Also, for a practical device, it is probably sufficient to control, say, $80-90 \%$ of the emission in such a way that the radiation losses into the remaining $10-20 \%$ of available modes become negligible. 
Although a lot can be learned from passive structures and their use as laser mirrors, the true test of the performance of a photonic microstructure is to place an active emitter inside and observe its radiation pattern. To our knowledge, only one such experiment has been successfully conducted with an optically pumped active layer ${ }^{14}$, partially because there is a very serious material problem: the most efficient light emitting materials we know, the III-V semiconductors, suffer from large non-radiative recombination at exposed surfaces, of which there are many in a photonic crystal. We have proposed a variety of techniques that could be used to overcome this problem, ranging from the more established methods of passivation, overgrowth and using materials with low surface recombination velocity to the more exotic techniques of selective oxidation and self-organised growth. Even silicon-based light emitters could be considered due to the virtual lack of surface recombination in this material. Some of the techniques are only available in a limited wavelength-range because they are dependent on a particular material property (e.g. island formation of In-compounds or selective oxidation of Al-compounds). The choice of what route to follow depends on the desired operating wavelength and the results obtained by further research in each of the areas. It seems clear, however, that photonic crystal light emitters will become a reality in the foreseable future, because they have fulfilled their promise so far and there is a range of possibilities to overcome the surface recombination problem.

\section{ACKNOWLEDGEMENTS}

TFK gratefully acknowledges support through a Royal Society University Research Fellowship. We also acknowledge considerable support from the Nanoelectronics Research Centre at Glasgow University, as well as colleagues in the Optoelectronics Research Group and elsewhere, in particular Henri Benisty, whose comments on the manuscript were invaluable. Some of the work described here was supported by the European contract ESPRIT SMILES No.8447 and some through DARPA, the Army Research Office and the National Science Foundation of the USA.

\section{REFERENCES}

1. E.M. Purcell, “Spontaneous emission probabilities at radiofrequencies”, Phys. Rev. 69, p.681, 1946.

2. E. Yablonovitch, T.J. Gmitter, R.D. Meade, A.M. Rappe, K.D. Brommer and J.D. Joannopoulos, "Donor and acceptor modes in photonic band-structure", Phys. Rev. Lett. 67, pp3380-3383, 1991.

3. T. F. Krauss, S. Brand, and R. M. De La Rue, "Two-dimensional photonic-bandgap structures operating at near-infrared wavelengths", Nature 383, pp.699-702, 1996.

4. T.F. Krauss, B.Voegele, C.R.Stanley, and R.M. De La Rue, "Waveguide microcavity based on photonic microstructures", IEEE Phot. Tech. Lett. 9, pp.176-178, 1997.

5. D. Labilloy, H. Benisty, C. Weisbuch, T.F. Krauss, R Houdre and U . Oesterle, "Use of guided spontaneous emission of a semiconductor to probe the optical properties of two-dimensional photonic crystals", Appl. Phys. Lett. 71, pp. 738$740,1997$.

6. J.S. Foresi, P.R. Villeneuve, J. Ferrera, E.R. Thoen, G. Steinmeyer, S. Fan, J. ED. Joannopoulos, L. C. Kimerling, H.I. Smith and E.P.Ippen, "Photonic bandgap microcavities in optical waveguides", to be published in Nature, November 1997.

7. D.Labilloy, H.Benisty, C.Weisbuch, T.F. Krauss, R.M.De La Rue, V. Bardinal, R. Houdre, U. Oesterle, D. Cassagne, and C. Jouanin, "Quantitative measurement of transmission, reflection and diffraction of two-dimensional photonic bandgap structures at near-infrared wavelengths", to be published in Phys. Rev. Lett., November 1997.

8. J. O'Brien, O. Painter, R. Lee, C.C. Cheng, A. Yariv and A. Scherer, "Lasers incorporating 2D photonic bandgap mirrors", El. Lett. 32, pp. 2243-2244, 1996.

9. D.L. Bullock, C.C. Shih and R.S. Margulies, "Photonic band-structure investigation of 2-dimensional bragg reflector mirrors for semiconductor-laser mode control”, J. Opt. Soc. B 10, pp.399-403, 1993.

10. T.F. Krauss, O. Painter, A. Scherer, J. S. Roberts and R. M. De La Rue, "Photonic microstructures as laser mirrors", accepted for publication in Optical Engineering, April 1998. 
11. T. Baba, M. Hamasaki, N. Watanabe, P. Kaewplung, A. Matsutani, T. Mukaihara, F. Koyama and K. Iga, "A novel short-cavity laser with deep-grating distributed bragg reflectors”, Jpn. J. Appl. Phys. Pt.1 35, pp.1390-1394, 1996.

12. H. DeNeve, J. Blondelle, P. Van Daele, P. Demeester, R. Baets and G. Borghs, "Recycling of guided mode light emission in planar microcavity light emitting diodes", Appl. Phys. Lett. 70, pp.799-801, 1997.

13. J.M. Gerard, D. Barrier, J.Y.Marzin, R. Kuszelewicz, L. Manin, E. Costard, V. Thierry-Mieg and T. Rivera, “Quantum boxes as active probes for photonic microstructures: The pillar microcavity case”, Appl. Phys. Lett. 69, pp.449-451, 1996.

14. J.M. Gerard et al., to be published.

15. L. Goldstein, F. Glas, J.Y.Marzin, M.N. Charasse and G. Leroux, "Growth by molecular-beam epitaxy and characterisation ofg InGaAs/GaAs strained-layer superlattices”, Appl. Phys. Lett. 47, pp.1099-1101, 1985.

16. M. Tabibazar, S, Kang, A.N. MacInnes, M.B Power, A.R. Barron, P.P. Jenkins and A.F. Hepp, "Electronic passivation of n-type and p-type GaAs using chemical-vapor deposited GaS”, Appl. Phys.Lett. 63, pp.625-627, 1993.

17. D.A. Harrison, R. Ares, S.P. Watkins, M.L.W. Thewalt, C.R. Bolognesi, D.J.S. Beckett and A.J. SpringThorpe, "Large photoluminescence enhancements from epitaxial GaAs passivated by postgrowth phosphidization", Appl. Phys. Lett. 70, pp.3275-3277, 1997.

18. Y. Rosenwaks, X.Li and T.J. Coutts, "Characterisation of heat-treated ITO/InP solar-cells", J.Vac.Sci.Tech. A15, pp.2354-2358, 1997.

19. E.Yablonovitch, H.M.Cox and T.J. Gmitter, "Nearly ideal electronic surfaces on naked In 0.53 Ga0.47As quantum wells", Appl. Phys.Lett. 52, pp1002-1004, 1988.

20. J.M. Gerard, "Prospects of high efficiency quantum boxes obtained by direct epitaxial growth", Confined Electrons and Photons, E. Burstein and C. Weisbuch (eds.), pp.357-381, Plenum, NewYork,1995.

21. J.A. Lott, N.N.Ledentsov, V.M.Ustinov, A.Yu. Egorov, A.E. Zhukov, P.S. Kop'ev, Zh. I. Alferov and D. Bimberg, "Vertical cavity lasers based on vertically coupled quantum dots", El. Lett. 33, pp.1150-1151, 1997.

22. J.M. Gerard, O. Cabrol and B. Sermage, "InAs quantum boxes: Highly efficient radiative traps for light emitting devices on Si”, Appl. Phys. Lett. 68, pp.3123-3125, 1996.

23. Y. Hayashi, T. Mukaihara, N. Hatori, N. Ohnoki, A. Matsutani, F. Koyama and K. Iga, "Lasing characteristics of lowthreshold oxide confinement InGaAs-GaAlAs vertical-cavity surface-emitting lasers”, IEEE Phot. Tech. Lett. 7, pp.12341236, 1995.

24. P.W. Evans and N. Holonyak, Jr., "Planar anisotropic oxidation of graded AlGaAs for high resolution vertical-wall current and light guiding in laser diodes”, Appl. Phys. Lett. 71, pp. 261-263, 1997.

25. A.G.Cullis, L.T.Canham and P.D.J. Calcott, "The structural and luminescence properties of porous silicon”, J.Appl. Phys. 82, pp.909-965, 1997.

26. L.C. Kimerling, K.D. Kolenbrander, J. Michel and J.Palm, "Light-emission from silicon", Sol. State Phys. 50, pp.333-381, 1997. 